\title{
A theory of resistivity in Kondo lattice materials: the memory function approach
}

\author{
Komal Kumari*, Raman Sharma* and Navinder Singh** \\ *Department of Physics, Himachal Pradesh University, \\ Shimla, India, Pin:171005. \\ ** Physical Research Laboratory, Ahmedabad, \\ India, Pin: 380009. ${ }^{* \dagger \ddagger}$
}

$17 / 12 / 2019$

\begin{abstract}
We theoretically analyse D.C. resistivity $(\rho)$ in the Kondo-lattice model using the powerful memory function approach. The complete temperature evolution of $\rho$ is investigated using the Wölfle-Götze expansion of the memory function. The resistivity in this model originates due to spin-flip magnetic scattering of conduction $s$-electron off the quasilocalized $d$ or $f$ electron spins. We find the famous resistivity upturn at lower temperature regime $\left(k_{B} T<<\mu_{d}\right)$, where $\mu_{d}$ is the effective chemical potential of $d$-electrons. In the high temperature regime $\left(\mu_{d}<<k_{B} T\right)$ we discover that $\rho \propto T^{\frac{3}{2}}$. The worked out theory is quantitatively compared with experimental data and reasonably good agreement is found.
\end{abstract}

\section{Introduction}

In heavy fermion materials such as $C e C u_{2} S i_{2}, C e C u_{2} G_{2}, U R u_{2} S i_{2}, U P d_{2} A l_{3}$ etc. [1, 2] and also in nano-scale granular aluminum [3] at sufficiently high temperatures $\left(T>T_{K}\right)$, where $T_{K}$ is the Kondo temperature) it becomes possible to divide the electronic system into two components: (1) mobile or conduction $s$-electrons, and (2) localized d or $\mathrm{f}$ moments. The localized moments leads to the typical curie susceptibility $\left(\chi \propto \frac{1}{T}\right)$ and the itinerant $s$-electrons provide the electrical conduction. As temperature is lowered the conduction electron spins start to quantum mechanically hybridize with the localized $d$ or $f$-moments. At sufficiently low temperature $T_{K}$, conduction electrons and localized $f$ moments form what is known as Kondo singlets. The process of hybridization is gradual one starting from higher temperature where $f$-moments are free, to very low temperature $\left(T<<T_{K}\right)$ where $f$-moments form spin singlets with conduction $s$ electrons. In this very low temperature regime emerges "hybridized electrons". These "hybridized electrons" are very heavy (their mass is many order of magnitude larger than free electron electron mass). Thus these systems are called heavy Fermion systems. It turns out that the Fermi volume contains both the conduction $s$-electrons and the "localized" $f$ or $d$ electrons in the $T<T_{K}$, and the superconducting transition happens in these "heavy electrons" [4].

\footnotetext{
* Email: sharmakomal611@gmail.com, komal.phyhpu@gmail.com

$\dagger$ Email: raman.sharma@hpuniv.ac.in,

‡Email: navinder@prl.res.in
} 
However, in the high temperature regime Fermi volume contains only the conduction electrons (not the localized $f$ or $d$-moments) [1, 2].

The current investigation is devoted to a different problem of electrical conduction in such systems. In the current investigation which is valid for $T>T_{K}$ we study the scattering of conduction $s$-electrons via the quasi-localized $f$-moments. Our aim is to calculate the temperature dependence of the electrical resistivity originating from magnetic scattering.

Resistivity from magnetic scattering is a well know phenomenon. The Kondo effect of resistivity minimum in materials containing magnetic impurities such as $A u F e$ is well studied[5, 6. 7]. It occurs due to spin flip scattering of conduction electrons via spin flips of localized magnetic impurity spin. J. Kondo explained it using second order perturbation theory [5, 6, 7]. In other words it takes into account the spin flip of the impurity and scattering electron as an intermediate state:

$$
\sum_{k^{\prime}} J\left(k \downarrow, \uparrow \rightarrow k^{\prime \prime} \uparrow, \downarrow\right) . J\left(k^{\prime \prime} \uparrow, \downarrow \rightarrow k^{\prime} \downarrow, \uparrow\right) \frac{\left(1-f_{k^{\prime \prime}}\right)}{\epsilon_{k}-\epsilon_{k^{\prime}}},
$$

where the factor $1-f_{k^{\prime \prime}}$ represents the probability that the state $\left|k^{\prime \prime}\right\rangle$ is empty. The above term represents the scattering of an electron with wavevector $k$ and spin state $|\downarrow\rangle$ and the impurity in spin state $|\uparrow\rangle$ into an intermediate state having electron with wavevector $k^{\prime \prime}$ but flipped spins for both the impurity and the electron. Then from this intermediate state electron scatters to a final state with wavevector $k^{\prime}$ with one more flips of electron and impurity spins, such that the spin states returns back to its original form. As is well known the resistivity due to above Kondo term scales as $\log (T)$ [5, 6, 7, 8]. However, this calculation does not capture full temperature evolution of resistivity. Our calculation using memory function formalism incorporates the above Kondo term and we analytically obtain the full temperature dependence of the resistivity including the high temperature behaviour $\left(\rho \propto T^{\frac{3}{2}}\right)$. In our calculation the coupling of $s$-electrons with quasi-localized $d$ or $f$-moments is taken to be the Kondo Coupling. We treat d or f electrons as quasi-localized instead of perfectly localized ones as considered in the standard Kondo problem. Perfect localization of $\mathrm{f}$ or $\mathrm{d}$ electrons occurs in the integer valence compounds (at half filling)[1]. Due to integer valence and strong onsite Coulomb repulsion (Hubbard U) double occupancy at a given site is prohibited. In our calculation we consider systems away from integer valence and $\mathrm{d}$ or $\mathrm{f}$ electrons are treated as quasi-localized, and they form a small Fermi surface ( refer to section 2 and appendix A). The coupling Hamiltonian is the Kondo lattice Hamiltonian also known in the literature as $s$ - $d$ Hamiltonian:

$$
H_{s d}=\frac{J}{N} \sum_{k^{\prime} k}\left\{a_{k^{\prime} \uparrow}^{\dagger} a_{k \downarrow} S^{-}\left(k^{\prime}-k\right)+a_{k^{\prime} \downarrow}^{\dagger} a_{k \uparrow} S^{+}\left(k^{\prime}-k\right)+\left(a_{k^{\prime} \uparrow}^{\dagger} a_{k \uparrow}-a_{k^{\prime} \downarrow}^{\dagger} a_{k \downarrow}\right) S^{z}\left(k^{\prime}-k\right)\right\}
$$

Here $a_{k^{\prime} \uparrow}^{\dagger} a_{k \downarrow}$ are the operators of $s$-electrons and $S^{-}\left(k^{\prime}-k\right)$ is the spin lowering operator of $d$-or $f$ electrons $\left(S^{-}(q)=\sum_{k} a_{k+q \downarrow}^{*} a_{k \uparrow}\right)$.

Another novelty of our calculation over the published calculations of electrical resistivity [9, 10, 11, 12, 13, 14, 15, 16 is that it is manifestly beyond the Relaxation Time Approximation (RTA) which is taken into account in the memory function formalism [19, 20] (our main tool in the current investigation) and full temperature evolution of the resistivity can be calculated whereas in the refs $[9,10,11,12,13,14,15,16]$ resistivity is calculated either using the variational solution of the Block-Boltzmann equation or the iterative approximate method [17, 19]. The problem with the Bloch-Boltzmann approach is that the full temperature evolution of resistivity is difficult to obtain analytically (only in low and hight temperature limits (say, with respect to the Debye temperature), the collision integral can be analytically simplified). Within the memory function formalism, we could analyse the full temperature evolution of resistivity 
rigorously and point out two regimes of interest: In the low temperature regime $\left(k_{B} T<<\mu_{d}\right)$, we find an upturn in the resistivity and in the high temperature regime $\left(k_{B} T>>\mu_{d}\right)$, we find that $\rho \propto T^{\frac{3}{2}}$. We compare our theory with the experimental data of ref. [3] and find good agreement.

\section{Computational procedure using MF formalism}

In Kubo's linear response theory, the dynamical conductivity is given by

$$
\sigma_{\mu \nu}(\omega)=V \int_{0}^{\infty} d t e^{i \omega t} \int_{0}^{\beta} d \lambda\left\langle J_{\mu}(-i \hbar \lambda) J_{\nu}(t)\right\rangle
$$

This is called the Kubo formula [18, 19, 20]. By using the Mori-Zwanzig projection operator technique the above Kubo formula can be rewritten in the following form[19, 20]

$$
\sigma_{\mu \nu}(z)=i \frac{\omega_{p}^{2}}{4 \pi} \frac{1}{z+M_{\mu \nu}(z)} .
$$

Here $M_{\mu \nu}(z)$ is called the memory function and $z$ is the complex frequency $(z=\omega+i \delta)$. Thus the problem of computation of the dynamical conductivity boils down to the computation of the memory function $M_{\mu \nu}(z)$. Within the Götze-Wölfle approach the memory function is computed using the equation of motion method and a perturbative expansion of the memory function. All the technical details are given in refs. [19, 20] here we outline the approach. It turns out that

$$
M(z) \simeq \frac{1}{z}\left(\frac{n e^{2}}{m}\right)\left[\left\langle\left\langle\dot{J}_{1} ; \dot{J}_{1}\right\rangle\right\rangle_{z}-\left\langle\left\langle\dot{J}_{1} ; \dot{J}_{1}\right\rangle\right\rangle_{0}\right]
$$

where

$$
\dot{J}_{1}=-\frac{i}{\hbar}\left[J_{1}, H\right]
$$

The total Hamiltonian is $H=H_{0}+H_{s d}$ and $H_{0}$ is the free electron unperturbed part and $H_{s d}$ is defined in eqn (2). The double brackets are defined as

$$
\left\langle\left\langle\hat{O}_{1} ; \hat{O}_{2}\right\rangle\right\rangle=i \frac{V}{\hbar} \int_{0}^{\infty} d t e^{i z t}\left\langle\left[\hat{O}_{1}(t), \hat{O}_{2}(0)\right]\right\rangle
$$

Here $\langle\ldots>$ means canonical ensemble average. The operator $\hat{O}(t)$ is in the Heisenberg representation $\hat{O}(t)=e^{i H t} \hat{O}(0) e^{-i H t}$. The current density operator is $J_{1}=\frac{1}{V} \sum_{k \sigma} e v_{k} a_{k \sigma}^{\dagger} a_{k \sigma}$ where $v_{k}=\frac{1}{\hbar} \frac{\partial \epsilon_{k}}{\partial k}$ and $V$ is the volume of the sample. With this information equation (6) takes the form:

$$
\begin{array}{r}
\dot{J}_{1}=-i \frac{e}{\hbar V} \sum_{l, \sigma} \sum_{k^{\prime} k}\left[v_{1}(l) a_{l \sigma}^{\dagger} a_{l \sigma}, \frac{J}{N} \sum_{k, k^{\prime}}\left\{a_{k^{\prime} \uparrow}^{\dagger} a_{k \downarrow} S^{-}\left(k^{\prime}-k\right)+a_{k^{\prime} \downarrow}^{\dagger} a_{k \uparrow} S^{+}\left(k^{\prime}-k\right)+\right.\right. \\
\left.\left.\left(a_{k^{\prime} \uparrow}^{\dagger} a_{k \uparrow}-a_{k^{\prime} \downarrow}^{\dagger} a_{k \downarrow}\right) S^{z}\left(k^{\prime}-k\right)\right\}\right]
\end{array}
$$

The current operator commutes with the unperturbed Hamiltonian, hence we are left with terms containing $H_{s d}$ which is treated as a perturbation. Using Leibniz's bracket rule $[a b, c]=$ $a\{b, c\}-\{a, c\} b$, the above expression reduces to

$$
\dot{J}_{1}=-\frac{i}{\hbar} \frac{e J}{N V} \sum_{k^{\prime} k}\left(v_{1}\left(k^{\prime}\right)-v_{1}(k)\right)\left(a_{k^{\prime} \uparrow}^{\dagger} a_{k \downarrow} S^{-}\left(k^{\prime}-k\right)+a_{k^{\prime} \downarrow}^{\dagger} a_{k \uparrow} S^{+}\left(k^{\prime}-k\right)\right) .
$$


Define the correlator $\phi(z)=\left\langle\left\langle\dot{J}_{1} ; \dot{J}_{1}\right\rangle\right\rangle$ :

$$
\begin{gathered}
\phi(z)=\frac{-e^{2} J^{2}}{N^{2} \hbar^{2} V^{2}} \sum_{k^{\prime} k} \sum_{p p^{\prime}}\left(v_{1}\left(k^{\prime}\right)-v_{1}(k)\right)\left(v_{1}(p)-v_{1}\left(p^{\prime}\right)\right)\left\langle\left\langle a_{k^{\prime} \uparrow}^{\dagger} a_{k \downarrow} S^{-}\left(k^{\prime}-k\right)+\right.\right. \\
\left.\left.a_{k^{\prime} \downarrow}^{\dagger} a_{k \uparrow} S^{+}\left(k^{\prime}-k\right) ; a_{p \uparrow}^{\dagger} a_{p^{\prime} \downarrow} S^{-}\left(p-p^{\prime}\right)+a_{p \downarrow}^{\dagger} a_{p^{\prime} \uparrow} S^{+}\left(p-p^{\prime}\right)\right\rangle\right\rangle .
\end{gathered}
$$

Then the memory function (15) can be written as $M(z) \simeq \frac{1}{z}\left(\frac{n e^{2}}{m}\right)(\phi(z)-\phi(0))$. This is called the Götze-Wölfle memory function approximation [19, 20]. Now for the computation of memory function we need to compute the correlator $(\phi(z))$

$$
\phi(z)=\left\langle\left\langle\dot{J}_{1} ; \dot{J}_{1}\right\rangle\right\rangle=i \frac{V}{\hbar} \int_{0}^{\infty} e^{i z t}\left\langle\left[\dot{J}_{1}(t) ; \dot{J}_{1}(0)\right]\right\rangle d t .
$$

The correlation function $\phi(z)$ can be simplified to

$$
\begin{aligned}
\phi(z)=\frac{-e^{2} J^{2}}{N^{2} \hbar^{2} V^{2}} \sum_{k^{\prime} k} & \sum_{p p^{\prime}}\left(v_{1}\left(k^{\prime}\right)-v_{1}(k)\right)\left(v_{1}(p)-v_{1}\left(p^{\prime}\right)\right)\left\{\left\langle\left\langle a_{k^{\prime} \uparrow}^{\dagger} a_{k \downarrow} S^{-}\left(k^{\prime}-k\right) ; a_{p \downarrow}^{\dagger} a_{p^{\prime} \uparrow} S^{+}\left(p-p^{\prime}\right)\right\rangle\right\rangle\right. \\
& \left.+\left\langle\left\langle a_{k^{\prime} \downarrow}^{\dagger} a_{k \uparrow} S^{+}\left(k^{\prime}-k\right) ; a_{p_{\uparrow}}^{\dagger} a_{p^{\prime} \downarrow} S^{-}\left(p-p^{\prime}\right)\right\rangle\right\rangle\right\}
\end{aligned}
$$

as the cross-terms of the form $\left\langle\left\langle a_{k^{\prime} \uparrow}^{\dagger} a_{k \downarrow} S^{-}\left(k^{\prime}-k\right) ; a_{p_{\uparrow}}^{\dagger} a_{p^{\prime} \downarrow} S^{-}\left(p-p^{\prime}\right)\right\rangle\right\rangle$ vanish [19, 20]. We separate the function $\phi(z)$ into two sub functions $\phi_{1}(z)$ and $\phi_{2}(z)$ for simplification. The first function takes the form:

$$
\begin{gathered}
\phi_{1}(z)=-i \frac{e^{2} J^{2}}{N^{2} \hbar^{3} V} \sum_{k^{\prime} k} \sum_{p p^{\prime}}\left(v_{1}\left(k^{\prime}\right)-v_{1}(k)\right)\left(v_{1}(p)-v_{1}\left(p^{\prime}\right)\right) \int_{0}^{\infty} d t e^{i z t}\left\langle\left[ a_{k^{\prime} \uparrow}^{\dagger}(t) a_{k \downarrow}(t) S^{-}\left(k^{\prime}-k, t\right),\right.\right. \\
\left.\left.a_{p \downarrow}^{\dagger} a_{p^{\prime} \uparrow} S^{+}\left(p-p^{\prime}\right)\right]\right\rangle
\end{gathered}
$$

It is to be noted the impurity and conduction electron spin flip terms of the form of eqn (2) are incorporated in the commutator in the above equation (13) that is $a_{k^{\prime} \uparrow}^{\dagger}(t) a_{k \downarrow}(t) S^{-}\left(k^{\prime}-\right.$ $k, t), a_{p \downarrow}^{\dagger} a_{p^{\prime} \uparrow} S^{+}\left(p-p^{\prime}\right)$ etc. We write the time dependence of operators explicitly as $a_{k^{\prime} \uparrow}^{\dagger}(t)=$ $e^{\frac{i k_{k^{\prime}} t}{\hbar}} a_{k^{\prime} \uparrow}^{\dagger}(0)$ for $s$-band mobile electronsi . For $d$ - band density operators we write $S^{-}\left(k^{\prime}-k, t\right)=$ $e^{-i \omega_{k^{\prime}-k} t} S^{-}\left(k^{\prime}-k, 0\right)$. In the present case $\hbar \omega_{k^{\prime}-k}$ represents the spin flip energy of an excitation of the quasi localized of $d$ or $f$ electrons. Dispersion of the magnetic excitation created by operators $S^{-}(q)$ and $S^{+}(q)$ is assumed to be of the form $\hbar \omega_{q} \propto q^{2}$ in the long wavelength limit which we use in the present calculation[10. Next on performing the time integration and applying anticommutating Leibniz ruleii to the Fermion operators in equation (13) we obtain

$$
\begin{aligned}
& \phi_{1}(z)=C_{1} \sum_{k^{\prime} k} \sum_{p p^{\prime}}\left(\frac{1}{\frac{\epsilon_{k^{\prime}}^{s}}{\hbar}-\frac{\epsilon_{k}^{s}}{\hbar}-\omega_{k^{\prime}-k}+z}\right)\left(v_{1}\left(k^{\prime}\right)-v_{1}(k)\right)\left(v_{1}(p)-v_{1}\left(p^{\prime}\right)\right)\left\langle\left(-a_{k^{\prime} \uparrow}^{\dagger} a_{k \downarrow} a_{p \downarrow}^{\dagger} a_{p^{\prime} \uparrow}\right)\right. \\
& {\left[S^{-}\left(k^{\prime}-k\right), S^{+}\left(p-p^{\prime}\right)\right]+\left\langle\left\{a_{k^{\prime} \uparrow}^{\dagger} a_{k \downarrow}, a_{p \downarrow}^{\dagger} a_{p^{\prime} \uparrow}\right\} S^{+}\left(p-p^{\prime}\right) S^{-}\left(k^{\prime}-k\right)\right\rangle .}
\end{aligned}
$$

\footnotetext{
${ }^{\mathrm{i}} \mathrm{As} a_{k^{\prime} \uparrow}^{\dagger}(t)$ is in the Heisenberg representation, it should be written as $a_{k^{\prime} \uparrow}^{\dagger}(t)=e^{\frac{i \epsilon_{k^{\prime}}^{T} t}{\hbar}} a_{k^{\prime} \uparrow}^{\dagger}(0)$, where $\epsilon_{k^{\prime}}^{T}$ is the eigenvalues of the total Hamiltonian $H=H_{0}+H_{s d}$. But we have replaced $\epsilon_{k^{\prime}}^{T}$ with $\epsilon_{k^{\prime}}$ which is the eigenvalue of the unperturbed or free electron Hamiltonian $H_{0}$. This approximation is valid as the perturbation $H_{s d}$ is assumed weaker (weak coupling limit of $J$ ) also refer to [20].

ii $\{a b, c d\}=a\{b, c\} d-a c\{b, d\}+\{a, c\} d b-c\{a, d\} b$
} 
Here $C_{1}=\frac{e^{2} J^{2}}{N^{2} \hbar^{3} V}$. We write $\left\langle a_{k^{\prime} \uparrow}^{\dagger} a_{k \downarrow} a_{p \downarrow}^{\dagger} a_{p^{\prime} \uparrow}\right\rangle=\left\langle a_{k^{\prime} \uparrow}^{\dagger}\left(\delta_{k p}-a_{p_{\downarrow}}^{\dagger} a_{k \downarrow}\right) a_{p^{\prime} \uparrow}\right\rangle$ and use bracket rule ${ }^{i i}$ to solve factor $\left\langle\left\{a_{k^{\prime} \uparrow}^{\dagger} a_{k \downarrow}, a_{p \downarrow}^{\dagger} a_{p^{\prime} \uparrow}\right\}\right\rangle$. On simplifying, using the properties of delta functions $\delta_{k, p}$ and $\delta_{k^{\prime}, p^{\prime}}$, we get:

$$
\begin{gathered}
\phi_{1}(z)=-C_{1} \sum_{k^{\prime} k}\left(\frac{1}{\frac{\epsilon_{k^{\prime}}^{s}}{\hbar}-\frac{\epsilon_{k}^{s}}{\hbar}-\omega_{k^{\prime}-k}+z}\right)\left(v_{1}\left(k^{\prime}\right)-v_{1}(k)\right)^{2}\left[f_{k^{\prime} \uparrow}^{s}\left(1-f_{k \downarrow}^{s}\right)\left\langle\left\{S^{-}\left(k^{\prime}-k\right), S^{+}\left(k-k^{\prime}\right)\right\}\right\rangle\right. \\
\left.+\left(f_{k^{\prime} \uparrow}^{s}-f_{k \downarrow}^{s}\right)\left\langle S^{+}\left(k-k^{\prime}\right) S^{-}\left(k^{\prime}-k\right)\right\rangle\right]
\end{gathered}
$$

Here $f_{k^{\prime} \uparrow}^{s}=\left\langle a_{k^{\prime} \uparrow}^{\dagger} a_{k^{\prime} \uparrow}\right\rangle$ is the Fermi function of the $s$-band electrons. The spin density operators of $d$-band transforms the expression (15) to [refer to appendix A]

$$
\begin{gathered}
\phi_{1}(z)=-C_{1} \sum_{k^{\prime} k}\left(\frac{1}{\frac{\epsilon_{k^{\prime}}^{s}}{\hbar}-\frac{\epsilon_{k}^{s}}{\hbar}-\omega_{k^{\prime}-k}+z}\right)\left(v_{1}\left(k^{\prime}\right)-v_{1}(k)\right)^{2}\left[f_{k^{\prime} \uparrow}^{s}\left(1-f_{k \downarrow}^{s}\right) \sum_{k_{d}, k_{d}^{\prime}}\left(f_{k_{d} \uparrow}^{d}-f_{k_{d^{\prime}} \downarrow}^{d}\right)-\right. \\
\left.\left(f_{k \downarrow}^{s}-f_{k^{\prime} \uparrow}^{s}\right) \sum_{k_{d}, k_{d}^{\prime}} f_{k_{d} \uparrow}^{d}\left(1-f_{k_{d^{\prime} \downarrow}}^{d}\right)\right] .
\end{gathered}
$$

Similary write $\phi_{2}(z)$ part from equation (12) :

$$
\begin{gathered}
\phi_{2}(z)=-\frac{e^{2} J^{2}}{N^{2} \hbar^{3} V^{2}} \sum_{k^{\prime} k} \sum_{p p^{\prime}}\left(v_{1}\left(k^{\prime}\right)-v_{1}(k)\right)\left(v_{1}(p)-v_{1}\left(p^{\prime}\right)\right)\left\langle\left\langle a_{k^{\prime} \downarrow}^{\dagger} a_{k \uparrow} S^{+}\left(k^{\prime}-k\right) ;\right.\right. \\
\left.\left.a_{p \uparrow}^{\dagger} a_{p^{\prime} \downarrow} S^{-}\left(p-p^{\prime}\right)\right\rangle\right\rangle .
\end{gathered}
$$

Again following the similar steps that are followed for the calculation of $\phi_{1}(z)$, we obtain expression for $\phi_{2}(z)$ as:

$$
\begin{gathered}
\phi_{2}(z)=-C_{1} \sum_{k^{\prime} k}\left(\frac{1}{\frac{\epsilon_{k^{\prime}}}{\hbar}-\frac{\epsilon_{k}^{s}}{\hbar}-\omega_{k^{\prime}-k}-z}\right)\left(v_{1}\left(k^{\prime}\right)-v_{1}(k)\right)^{2}\left[f_{k^{\prime} \uparrow}^{s}\left(1-f_{k \downarrow}^{s}\right) \sum_{k_{d}, k_{d}^{\prime}}\left(f_{k_{d} \uparrow}^{d}-f_{k_{d}^{\prime} \downarrow}^{d}\right)-\right. \\
\left.\left(f_{k \downarrow}^{s}-f_{k^{\prime} \uparrow}^{s}\right) \sum_{k_{d}, k_{d}^{\prime}} f_{k_{d} \uparrow}^{d}\left(1-f_{k_{d^{\prime}} \downarrow}^{d}\right)\right] .
\end{gathered}
$$

We drop the spin notation in Fermi functions as there is no Zeeman splitting(no external and internal magnetic fields present). The total $\phi(z)$ takes the form:

$$
\begin{array}{r}
\phi(z)=-\frac{e^{2} J^{2}}{N^{2} \hbar^{3} V} \sum_{k^{\prime} k}\left(v_{1}\left(k^{\prime}\right)-v_{1}(k)\right)^{2}\left\{f_{k^{\prime}}^{s}\left(1-f_{k}^{s}\right) \sum_{k_{d}, k_{d}^{\prime}}\left(f_{k_{d}}^{d}-f_{k_{d}^{\prime}}^{d}\right)-\left(f_{k}^{s}-f_{k^{\prime}}^{s}\right) \times\right. \\
\left.\sum_{k_{d}, k_{d}^{\prime}} f_{k_{d}}^{d}\left(1-f_{k_{d}^{\prime}}^{d}\right)\right\}\left[\frac{1}{\frac{\epsilon_{k^{\prime}}}{\hbar}-\frac{\epsilon_{k}}{\hbar}-\omega_{k^{\prime}-k}+z}+\frac{1}{\frac{\epsilon_{k^{\prime}}}{\hbar}-\frac{\epsilon_{k}}{\hbar}-\omega_{k^{\prime}-k}-z}\right] .
\end{array}
$$

\section{Computation of the Memory Function in the DC limit}

Our aim is to determine the dynamical conductivity $\sigma(z)$ that depends on the Memory function, therefore writing $\phi(z)$ in terms of $M(z)$ using formula $M(z)=\frac{1}{z} \frac{m}{n e^{2}}(\phi(z)-\phi(0))$, we obtain 


$$
\begin{aligned}
M(z)= & -\frac{J^{2} m}{N^{2} \hbar^{3} n V \omega} \sum_{k^{\prime} k}\left(v_{1}\left(k^{\prime}\right)-v_{1}(k)\right)^{2}\left\{f_{k^{\prime}}^{s}\left(1-f_{k}^{s}\right) \sum_{k_{d}, k_{d}^{\prime}}\left(f_{k_{d}}^{d}-f_{k_{d}^{\prime}}^{d}\right)-\left(f_{k}^{s}-f_{k^{\prime}}^{s}\right) \sum_{k_{d}, k_{d}^{\prime}} f_{k_{d}}^{d}\left(1-f_{k_{d}^{\prime}}^{d}\right)\right\} \\
& {\left[\frac{1}{\frac{\epsilon_{k^{\prime}}}{\hbar}-\frac{\epsilon_{k}}{\hbar}-\omega_{k^{\prime}-k}+z}+\frac{1}{\frac{\epsilon_{k^{\prime}}}{\hbar}-\frac{\epsilon_{k}}{\hbar}-\omega_{k^{\prime}-k}-z}-\frac{1}{\frac{\epsilon_{k^{\prime}}}{\hbar}-\frac{\epsilon_{k}}{\hbar}-\omega_{k^{\prime}-k}}-\frac{1}{\frac{\epsilon_{k^{\prime}}}{\hbar}-\frac{\epsilon_{k}}{\hbar}-\omega_{k^{\prime}-k}}\right] }
\end{aligned}
$$

Where $M(z)=M(\omega \pm i 0)=M^{\prime}(\omega) \pm i M^{\prime \prime}(\omega)$. Here we are interested in the imaginary part of the memory function [19, 20]. The use of identity $\lim _{\eta \rightarrow 0} \frac{1}{a \mp i \eta}=\mathfrak{P}\left(\frac{1}{a}\right) \pm i \pi \delta(a)$ transforms the expression (20) iii into delta function form. On comparing imaginary part of the above expression, we get

$$
\begin{aligned}
M^{\prime \prime}(\omega)= & \frac{J^{2} m \pi}{N^{2} \hbar^{3} n V \omega} \sum_{k^{\prime} k}\left(v_{1}\left(k^{\prime}\right)-v_{1}(k)\right)^{2}\left\{f_{k^{\prime}}^{s}\left(1-f_{k}^{s}\right) \sum_{k_{d}, k_{d}^{\prime}}\left(f_{k_{d}}^{d}-f_{k_{d}^{\prime}}^{d}\right)-\right. \\
& \left.\left(f_{k}^{s}-f_{k^{\prime}}^{s}\right) \sum_{k_{d}, k_{d}^{\prime}} f_{k_{d}}^{d}\left(1-f_{k_{d}^{\prime}}^{d}\right)\right\}\left[\delta\left(\frac{\epsilon_{k^{\prime}}}{\hbar}-\frac{\epsilon_{k}}{\hbar}-\omega_{k^{\prime}-k}+\omega\right)-\delta\left(\frac{\epsilon_{k^{\prime}}}{\hbar}-\frac{\epsilon_{k}}{\hbar}-\omega_{k^{\prime}-k}-\omega\right)\right] .
\end{aligned}
$$

Using the momentum conservation $\vec{k}^{\prime}-\vec{k}=\vec{k}_{d}^{\prime}-\vec{k}_{d}=\vec{q}$, write $\vec{k}^{\prime}$ and $\vec{k}_{d}^{\prime}$ in terms of $\vec{k}+\vec{q}$ and $\vec{k}_{d}+\vec{q}$. Also write $\left(v_{1}\left(k^{\prime}\right)-v_{1}(k)\right)^{2}=\frac{\hbar^{2}}{m^{2}}\left(\vec{k}^{\prime}-\vec{k}\right)^{2}$. To deal with the magnitude of $\left(\vec{k}^{\prime}-\vec{k}\right)$, i.e. $\left|\overrightarrow{k^{\prime}}-\vec{k}\right|$ insert an integral $d q \delta\left(\vec{q}-\left|\overrightarrow{k^{\prime}}-\vec{k}\right|\right)$ over $q$ into equation (21) which simplify the calculation greatly. Using the spatial isotropy in the present free electron case we can write $v^{2}=\left(v_{x}^{2}+v_{y}^{2}+v_{z}^{2}\right)=3 v_{x}^{2}$. Converting sums into integrals for $k$ and $k^{\prime}$ using $\frac{1}{V} \sum \rightarrow \int \frac{d^{3} k}{(2 \pi)^{3}}$, the above equation can be written as

$$
\begin{gathered}
M^{\prime \prime}(\omega)=\frac{J^{2} \pi V}{3 N^{2} m n} \int_{0}^{\infty} \frac{d q}{\omega} q^{2} \int_{0}^{\infty} \frac{d^{3} k}{(2 \pi)^{3}} \int_{0}^{\infty} \frac{d^{3} k^{\prime}}{(2 \pi)^{3}} \delta\left(\vec{q}-\left|\vec{k}^{\prime}-\vec{k}\right|\right) F\left(f_{k}^{s}, f_{k^{\prime}}^{s}, f_{k_{d}}^{d}, f_{k_{d}^{\prime}}^{d}\right) \\
{\left[\delta\left(\epsilon_{k+q}-\epsilon_{k}-\hbar \omega_{q}+\hbar \omega\right)-\delta\left(\epsilon_{k+q}-\epsilon_{k}-\hbar \omega_{q}-\hbar \omega\right)\right] .}
\end{gathered}
$$

Here, we write $F\left(f_{k}^{s}, f_{k^{\prime}}^{s}, f_{k_{d}}^{d}, f_{k_{d}^{\prime}}^{d}\right)$ as short hand notation for Fermi distribution function inside the curly braces. Write $\int d^{3} k=4 \pi \int k^{2} d k, \int d^{3} k^{\prime}=2 \pi \int k^{2} d k^{\prime} \int_{0}^{\pi} \sin \theta d \theta$ (take $k$ as pointing along the $z$-direction). Therefore $M^{\prime \prime}(\omega)$ takes the form

$$
\begin{aligned}
M^{\prime \prime}(\omega)= & \frac{2 J^{2} \pi V}{3 N^{2} m n} \frac{(2 \pi)^{2}}{(2 \pi)^{6}} \int_{0}^{\infty} \frac{d q}{\omega} q^{2} \int_{0}^{\infty} k^{2} d k \int_{0}^{\infty} k^{\prime 2} d k^{\prime} \int_{0}^{\pi} \sin \theta d \theta \delta\left(q-\sqrt{\left(k^{\prime 2}+k^{2}-2 k^{\prime} k \cos \theta\right)}\right) \\
& \sum_{k_{d}, k_{d}^{\prime}} F\left(f_{k}^{s}, f_{k^{\prime}}^{s}, f_{k_{d}}^{d}, f_{k_{d}^{\prime}}^{d}\right)\left[\delta\left(\epsilon_{k+q}-\epsilon_{k}-\hbar \omega_{q}+\hbar \omega\right)-\delta\left(\epsilon_{k+q}-\epsilon_{k}-\hbar \omega_{q}-\hbar \omega\right)\right]
\end{aligned}
$$

To simplify further, we shift momentum integral variables into energy variables $k^{2}=\frac{2 m \epsilon}{\hbar^{2}}$ and $d k=\frac{1}{\hbar} \sqrt{\frac{m}{2 \epsilon}} d \epsilon$. On writing $\epsilon_{k}$ as $\epsilon$ and $\epsilon_{k^{\prime}}$ as $\epsilon^{\prime}$ changes the expression to

$$
\begin{aligned}
M^{\prime \prime}(\omega)=\frac{2 J^{2} V m^{2}}{3 N^{2} \hbar^{6} n} \frac{1}{(2 \pi)^{3}} \int_{0}^{\infty} \frac{d q q^{2}}{\omega} \int_{0}^{\infty} \sqrt{\epsilon} d \epsilon \int_{0}^{\infty} \sqrt{\epsilon^{\prime}} d \epsilon^{\prime} \int_{0}^{\pi} \sin \theta d \theta \times \\
\delta\left(q-\sqrt{2 m} \sqrt{\left(\epsilon^{\prime}+\epsilon-2 \sqrt{\epsilon^{\prime} \epsilon} \cos \theta\right)}\right) \sum_{k_{d}} F\left(f_{k}^{s}, f_{k^{\prime}}^{s}, f_{k_{d}}^{d}, f_{k_{d}^{\prime}}^{d}\right) \times \\
{\left[\delta\left(\epsilon_{k+q}-\epsilon_{k}-\hbar \omega_{q}+\hbar \omega\right)-\delta\left(\epsilon_{k+q}-\epsilon_{k}-\hbar \omega_{q}-\hbar \omega\right)\right] . }
\end{aligned}
$$

${ }^{\mathrm{iii}} \lim _{\eta \rightarrow 0} \frac{1}{\epsilon_{k^{\prime}}-\epsilon_{k}-\omega_{q}+\omega \pm i \eta}=\mathfrak{P}\left(\frac{1}{\epsilon_{k^{\prime}}-\epsilon_{k}-\omega_{q}+\omega}\right) \mp i \pi \delta\left(\epsilon_{k^{\prime}}-\epsilon_{k}-\omega_{q}+\omega\right)$ 
On performing the $\theta$ integral the above expression (appendix B) reduces to the form

$$
\begin{aligned}
M^{\prime \prime}(\omega)= & \frac{1}{4 \pi^{3}} \frac{J^{2} V m^{2}}{3 N^{2} \hbar^{6} n} \int_{0}^{q_{D}} \frac{d q q^{2} q}{k_{s}^{2} \omega} \int_{0}^{\infty} \sqrt{\epsilon} d \epsilon \int_{0}^{\infty} d \epsilon^{\prime} \sqrt{\epsilon^{\prime}}\left\{f_{k+q}^{s}\left(1-f_{k}^{s}\right) \sum_{k_{d}}\left(f_{k_{d}}^{d}-f_{k_{d}+q}^{d}\right)-\right. \\
& \left.\left(f_{k}^{s}-f_{k+q}^{s}\right) \sum_{k_{d}} f_{k_{d}}^{d}\left(1-f_{k_{d}+q}^{d}\right)\right\}\left[\delta\left(\epsilon_{k+q}-\epsilon_{k}-\hbar \omega_{q}+\hbar \omega\right)-\delta\left(\epsilon_{k+q}-\epsilon_{k}-\hbar \omega_{q}-\hbar \omega\right)\right] .
\end{aligned}
$$

By using $f(x) \delta(x-a)=f(a) \delta(x-a)$ we remove $\epsilon_{k^{\prime}}$ from the Fermi functions and integrate over $\epsilon_{k^{\prime}}$ which we simply write $\epsilon^{\prime}$

$$
\begin{gathered}
M^{\prime \prime}(\omega)=p_{0} \int_{0}^{q_{D}} d q q^{3} \int_{0}^{\infty} d \epsilon \frac{\sqrt{\epsilon}}{\omega}\left[\sqrt { \epsilon _ { k } + \hbar \omega _ { q } - \hbar \omega } \sum _ { k _ { d } } \left\{f^{s}\left(\epsilon_{k}+\hbar \omega_{q}-\hbar \omega\right)\left(1-f^{s}\left(\epsilon_{k}\right)\right) \times\right.\right. \\
\left.\sum_{k_{d}}\left(f^{d}\left(\epsilon_{k_{d}}\right)-f^{d}\left(\epsilon_{k_{d}+q}\right)\right)-\left(f^{s}\left(\epsilon_{k}\right)-f^{s}\left(\epsilon_{k}+\hbar \omega_{q}-\hbar \omega\right)\right) \sum_{k_{d}} f^{d}\left(\epsilon_{k_{d}}\right)\left(1-f^{d}\left(\epsilon_{k_{d}+q}\right)\right)\right\}- \\
\sqrt{\epsilon_{k}+\hbar \omega_{q}+\hbar \omega}\left\{f^{s}\left(\epsilon_{k}+\hbar \omega_{q}+\hbar \omega\right)\left(1-f^{s}\left(\epsilon_{k}\right)\right) \sum_{k_{d}}\left(f^{d}\left(\epsilon_{k_{d}}\right)-f^{d}\left(\epsilon_{k_{d}+q}\right)\right)-\right. \\
\left.\left.\left(f^{s}\left(\epsilon_{k}\right)-f^{s}\left(\epsilon_{k}+\hbar \omega_{q}+\hbar \omega\right)\right) \sum_{k_{d}} f^{d}\left(\epsilon_{k_{d}}\right)\left(1-f^{d}\left(\epsilon_{k_{d}+q}\right)\right)\right\}\right]
\end{gathered}
$$

where the prefactors $p_{0}=\frac{1}{4 \pi^{3}} \frac{J^{2} V m^{2}}{3 N^{2} \hbar^{6} n q_{s}^{2}}$. Define $\epsilon_{k}^{s}=\epsilon, f_{d}^{1}=\sum_{k_{d}}\left(f^{d}\left(\epsilon_{k_{d}}\right)-f^{d}\left(\epsilon_{k_{d}+q}\right)\right)$ and $f_{d}^{2}=\sum_{k_{d}} f^{d}\left(\epsilon_{k_{d}}\right)\left(1-f^{d}\left(\epsilon_{k_{d}+q}\right)\right)$. With these definitions, we have

$$
\begin{gathered}
M^{\prime \prime}(\omega, T)=p_{0} \int_{0}^{q_{D}} d q q^{3}\left\{\int_{0}^{\infty} d \epsilon \frac{\sqrt{\epsilon}}{\omega}[\underbrace{\sqrt{\epsilon+\hbar \omega_{q}-\hbar \omega} f^{s}\left(\epsilon+\hbar \omega_{q}-\hbar \omega\right)\left(1-f^{s}(\epsilon)\right)}_{\operatorname{term}\left(T_{1}\right)}-\right. \\
\underbrace{\sqrt{\epsilon+\hbar \omega_{q}+\hbar \omega} \underbrace{s}_{\operatorname{term}\left(T_{2}\right)}\left(\epsilon+\hbar \omega_{q}+\hbar \omega\right)\left(1-f^{s}(\epsilon)\right)]}_{\operatorname{term}\left(T_{2}\right)} f_{d}^{1}(q)+ \\
\int_{0}^{\infty} d \epsilon \frac{\sqrt{\epsilon}}{\omega} \underbrace{\left[\sqrt{\epsilon+\hbar \omega_{q}+\hbar \omega}\left(f^{s}(\epsilon)-f^{s}\left(\epsilon+\hbar \omega_{q}+\hbar \omega\right)\right)\right.}_{\operatorname{term}\left(T_{3}\right)} \\
-\underbrace{\left.\sqrt{\epsilon+\hbar \omega_{q}-\hbar \omega}\left(f^{s}(\epsilon)-f^{s}\left(\epsilon+\hbar \omega_{q}-\hbar \omega\right)\right)\right]}_{\operatorname{term}\left(T_{4}\right)} f_{d}^{2}(q)\} .
\end{gathered}
$$

This is important general expression of imaginary part of the Memory Function, which is valid for all frequencies and all temperature regimes. In what follows, we analyze the above expression in the D.C. limit and study the temperature dependence of the imaginary part of the memory function. For performing the limit $\omega \rightarrow 0$, we rewrite the main result (equation 27) in the 
following way:

$$
\begin{aligned}
M^{\prime \prime}(\omega)= & p_{0} \int_{0}^{q_{D}} d q q^{3}\left[\int_{0}^{\infty} d \epsilon \sqrt{\epsilon} \times\right. \\
& (\underbrace{\frac{T e 1}{\sqrt{\epsilon+\hbar \omega_{q}+\hbar \omega}-\sqrt{\epsilon+\hbar \omega_{q}-\hbar \omega}}}_{\frac{\sqrt{\epsilon+\hbar \omega_{q}-\hbar \omega} f^{s}\left(\epsilon+\hbar \omega_{q}-\hbar \omega\right)-\sqrt{\epsilon+\hbar \omega_{q}+\hbar \omega} f^{s}\left(\epsilon+\hbar \omega_{q}+\hbar \omega\right)}{\omega}}) f^{s}(\epsilon) f_{d}^{2}(q)+ \\
& \left.\times\left(1-f^{s}(\epsilon)\right) f_{d}^{1}(q)+\int_{0}^{\infty} d \epsilon \sqrt{\epsilon}(\underbrace{\left.\frac{\sqrt{\epsilon+\hbar \omega_{q}+\hbar \omega} f^{s}}{\omega}+\hbar \omega_{q}+\hbar \omega\right)}_{022}) f_{d}^{2}(q)\right] .
\end{aligned}
$$

On performing the limit $\omega \rightarrow 0$ for term (Te1) we have

$$
\begin{aligned}
&\left.\frac{\partial T e 1}{\partial \omega}\right|_{\omega=0}=-\hbar \frac{f^{s}\left(\epsilon+\hbar \omega_{q}-\hbar \omega\right)}{2 \sqrt{\epsilon+\hbar \omega_{q}-\hbar \omega}}+\left.\sqrt{\epsilon+\hbar \omega_{q}-\hbar \omega} \frac{\partial f^{s}\left(\epsilon+\hbar \omega_{q}-\hbar \omega\right)}{\partial \omega}\right|_{\omega=0}-\hbar \frac{f^{s}\left(\epsilon+\hbar \omega_{q}+\hbar \omega\right)}{2 \sqrt{\epsilon+\hbar \omega_{q}+\hbar \omega}} \\
&-\left.\sqrt{\epsilon+\hbar \omega_{q}+\hbar \omega} \frac{\partial f^{s}\left(\epsilon+\hbar \omega_{q}+\hbar \omega\right)}{\partial \omega}\right|_{\omega=0} \\
&=-\hbar \frac{f^{s}\left(\epsilon+\hbar \omega_{q}\right)}{\sqrt{\epsilon+\hbar \omega_{q}}}+2 \hbar \beta \sqrt{\epsilon+\hbar \omega_{q}} \frac{e^{\beta\left(\epsilon+\hbar \omega_{q}-\mu_{s}\right)}}{\left(e^{\beta\left(\epsilon+\hbar \omega_{q}-\mu_{s}\right)}+1\right)^{2}}
\end{aligned}
$$

and for term (Te2), we have

$$
\left.\frac{\partial T e 2}{\partial \omega}\right|_{\omega=0}=\left.\frac{\hbar}{2 \sqrt{\epsilon+\hbar \omega_{q}+\hbar \omega}}\right|_{\omega=0}+\left.\frac{\hbar}{2 \sqrt{\epsilon+\hbar \omega_{q}-\hbar \omega}}\right|_{\omega=0}=\frac{\hbar}{\sqrt{\epsilon+\hbar \omega_{q}}} .
$$

Substituting the above expressions into eqn (28) we obtain the memory function in the D.C. limit

$$
\begin{gathered}
M^{\prime \prime}(T)=p_{0} \hbar \int_{0}^{q_{D}} d q q^{3}\left[\int_{0}^{\infty} d \epsilon \sqrt{\epsilon}\left(-\frac{f^{s}\left(\epsilon+\hbar \omega_{q}\right)}{\sqrt{\epsilon+\hbar \omega_{q}}}+2 \beta \sqrt{\epsilon+\hbar \omega_{q}} \frac{e^{\beta\left(\epsilon+\hbar \omega_{q}-\mu_{s}\right)}}{\left(e^{\beta\left(\epsilon+\hbar \omega_{q}-\mu_{s}\right)}+1\right)^{2}}\right) \times\right. \\
\left(1-f^{s}(\epsilon)\right) f_{d}^{1}(q)+\int_{0}^{\infty} \frac{d \epsilon \sqrt{\epsilon}}{\sqrt{\epsilon+\hbar \omega_{q}}} f^{s}(\epsilon) f_{d}^{2}(q)+\int_{0}^{\infty} d \epsilon \sqrt{\epsilon}\left(-\frac{f^{s}\left(\epsilon+\hbar \omega_{q}\right)}{\sqrt{\epsilon+\hbar \omega_{q}}}+\right. \\
\left.\left.2 \beta \sqrt{\epsilon+\hbar \omega_{q}} \frac{e^{\beta\left(\epsilon+\hbar \omega_{q}-\mu_{s}\right)}}{\left(e^{\beta\left(\epsilon+\hbar \omega_{q}-\mu_{s}\right)}+1\right)^{2}}\right) f_{d}^{2}(q)\right]
\end{gathered}
$$

There are a couple of reasonable assumptions which we would like to use to simplify the above expression: (1) The above expression can be simplified as $k_{B} T<<\mu_{s}$ (chemical potential for s-electrons) at temperature of interest $\left(\mu_{s} \simeq 10 \mathrm{eV}\right.$ and room temperature is $\left.\sim \frac{1}{40} \mathrm{eV}\right)$. (2) $\hbar \omega_{q}<<\mu_{s}$, that is, the energy scale of magnetic excitation (which is in meV) is much less than $\mu_{s}(\sim 10 \mathrm{eV})$. On implementing the second assumption in the Fermi function $f^{s}\left(\epsilon+\hbar \omega_{q}\right)=$ $\frac{1}{e^{\beta\left(\epsilon+\hbar \omega_{q}-\mu_{s}\right)}+1}$ lead to $f^{s}(\epsilon)$ and the above expression becomes

$$
\begin{aligned}
M^{\prime \prime}(T)=p_{0} \hbar & {\left[2 \beta \int_{0}^{q_{D}} d q q^{3} \int_{0}^{\infty} d \epsilon \sqrt{\epsilon} \sqrt{\epsilon+\hbar \omega_{q}} f^{s}(\epsilon)\left(1-f^{s}(\epsilon)\right)\left[\left(1-f^{s}(\epsilon)\right) f_{d}^{1}(q)+f_{d}^{2}(q)\right]\right.} \\
& \left.-\int_{0}^{q_{d}} d q q^{3} \int_{0}^{\infty} d \epsilon \sqrt{\epsilon} \frac{f^{s}(\epsilon)}{\sqrt{\epsilon+\hbar \omega_{q}}}\left(1-f^{s}(\epsilon)\right) f_{d}^{1}(q)\right]
\end{aligned}
$$


Next, on implementing the first assumption $k_{B} T<<\mu_{s}$, we notice that factors of the form $f^{s}(\epsilon)\left(1-f^{s}(\epsilon)\right)$ are approximately like delta functions peaking at $\mu_{s}$. Thus the relevant range of the $\epsilon$ is around $\mu_{s}$ with width of order $k_{B} T$. Observing this fact we can write $\sqrt{\epsilon+\hbar \omega_{q}} \simeq \sqrt{\epsilon}$ as $\hbar \omega_{q}<<\mu_{s}$ :

$$
\begin{aligned}
& M^{\prime \prime}(T)= p_{0} \hbar\left[2 \beta \int_{0}^{\infty} d \epsilon \sqrt{\epsilon} \sqrt{\epsilon} f^{s}(\epsilon)\left(1-f^{s}(\epsilon)\right)(\left(1-f^{s}(\epsilon)\right) \underbrace{\int_{0}^{q_{d}} d q q^{3} f_{d}^{1}(q)}_{I_{1}(T)}+\right. \\
&\underbrace{\int_{0}^{q_{d}} d q q^{3} f_{d}^{2}(q)}_{I_{2}(T)})-\int_{0}^{\infty} d \epsilon \sqrt{\epsilon} \frac{f^{s}(\epsilon)}{\sqrt{\epsilon}}\left(1-f^{s}(\epsilon)\right) \underbrace{\int_{0}^{q_{d}} d q q^{3} f_{d}^{1}(q)}_{I_{1}(T)}] \\
& M^{\prime \prime}(T)= p_{0} \hbar\left[2 \beta \int_{0}^{\infty} d \epsilon \epsilon f^{s}(\epsilon)\left(1-f^{s}(\epsilon)\right)\left(\left(1-f^{s}(\epsilon)\right) I_{1}(T)+I_{2}(T)\right)-\right. \\
& \int_{0}^{\infty} d \epsilon f^{s}(\epsilon)\left(1-f^{s}(\epsilon)\right) I_{1}(T)
\end{aligned}
$$

Integrals over $\epsilon$ can be performed using the properties of delta functions $f^{s}(\epsilon)\left(1-f^{s}(\epsilon)\right) \simeq$ $\frac{1}{\beta} \delta\left(\epsilon-\mu_{s}\right)$ :

$$
M^{\prime \prime}(T)=\frac{p_{0} \hbar}{\beta}\left[\left(\beta \mu_{s}-1\right) I_{1}(T)+2 \beta \mu_{s} I_{2}(T)\right]
$$

As $\beta \mu_{s}>>1$, we get

$$
M^{\prime \prime}(T)=p_{0} \hbar \mu_{s}\left[I_{1}(T)+2 I_{2}(T)\right]
$$

where

$$
I_{1}(T)=\int_{0}^{q_{D}} d q q^{3} f_{d}^{1}(q),
$$

and

$$
I_{2}(T)=\int_{0}^{q_{D}} d q q^{3} f_{d}^{2}(q) .
$$

The above simplified expression (eqn 36) is our main result in the DC limit. Our next aim is to reduce the expression for $I_{1}(T)$ and $I_{2}(T)$. For this we take the long wavelength approximation (small $q$ expansion). It can be shown (refer to Appendix C) that $f_{d}^{1}\left(\epsilon_{d}\right)$ in long wavelength limit $q \rightarrow 0$ can be written as

$$
f_{d}^{1}\left(\epsilon_{d}\right)=\frac{V q^{2}}{4 \pi^{2}} \frac{\sqrt{2 m}}{\hbar}\left[\beta \int_{0}^{\infty} \frac{d \epsilon_{d} \sqrt{\epsilon_{d}} e^{\beta\left(\epsilon_{d}-\mu_{d}\right)}}{\left(e^{\beta\left(\epsilon_{d}-\mu_{d}\right)}+1\right)^{2}}+\frac{2}{3} \beta^{2} \int_{0}^{\infty} \frac{d \epsilon_{d} \epsilon_{d}^{\frac{3}{2}} e^{\beta\left(\epsilon_{d}-\mu_{d}\right)}}{\left(e^{\beta\left(\epsilon_{d}-\mu_{d}\right)}+1\right)^{2}}-\frac{4}{3} \beta^{2} \int_{0}^{\infty} \frac{d \epsilon \epsilon^{\frac{3}{2}} e^{2 \beta\left(\epsilon_{d}-\mu_{d}\right)}}{\left(e^{\beta\left(\epsilon_{d}-\mu_{d}\right)}+1\right)^{3}}\right]
$$

on substituting the above expression of $f_{d}^{1}\left(\epsilon_{d}\right)$ into eqn (37) we get

$$
\begin{aligned}
I_{1}(T)=\frac{q_{D}^{6}}{6} \frac{V}{4 \pi^{2}} & \frac{\sqrt{2 m}}{\hbar}\left[\beta \int_{0}^{\infty} \frac{d \epsilon_{d} \sqrt{\epsilon_{d}} e^{\beta\left(\epsilon_{d}-\mu_{d}\right)}}{\left(e^{\beta\left(\epsilon_{d}-\mu_{d}\right)}+1\right)^{2}}+\frac{2}{3} \beta^{2} \int_{0}^{\infty} \frac{d \epsilon_{d} \epsilon_{d}^{\frac{3}{2}} e^{\beta\left(\epsilon_{d}-\mu_{d}\right)}}{\left(e^{\beta\left(\epsilon_{d}-\mu_{d}\right)}+1\right)^{2}}\right. \\
& \left.-\frac{4}{3} \beta^{2} \int_{0}^{\infty} \frac{d \epsilon \epsilon^{\frac{3}{2}} e^{2 \beta\left(\epsilon_{d}-\mu_{d}\right)}}{\left(e^{\beta\left(\epsilon_{d}-\mu_{d}\right)}+1\right)^{3}}\right]
\end{aligned}
$$


Similarly $f_{d}^{2}$ can be simplified (refer to appendix D) and the simplified expression of $f_{d}^{2}$ can be substituted into eqn (38). The result is

$$
I_{2}(T)=\frac{V}{(2 \pi)^{2}} \frac{(2 m)^{\frac{3}{2}}}{\hbar^{3}} \int_{0}^{q_{D}} d q q^{3} \int_{0}^{\infty} \frac{d \epsilon_{d} \sqrt{\epsilon_{d}} e^{\beta\left(\epsilon_{d}-\mu_{d}\right)}}{\left(e^{\beta\left(\epsilon_{d}-\mu_{d}\right)}+1\right)^{2}}+I_{1}(T)
$$

on substituting expressions of $I_{1}(T)$ and $I_{2}(T)$ into eqn (36) we have

$$
\begin{aligned}
& M^{\prime \prime}(T)= \frac{1}{12 \pi^{3}} \frac{J^{2} V^{2} m^{2}}{N^{2} \hbar^{5} n q_{s}^{2}} \mu_{s}\left\{\frac { 3 q _ { D } ^ { 6 } } { 6 } \frac { 1 } { 4 \pi ^ { 2 } } \frac { \sqrt { 2 m } } { \hbar } \left(\beta \int_{0}^{\infty} \frac{d \epsilon_{d} \sqrt{\epsilon_{d}} e^{\beta\left(\epsilon_{d}-\mu_{d}\right)}}{\left(e^{\beta\left(\epsilon_{d}-\mu_{d}\right)}+1\right)^{2}}+\frac{2}{3} \beta^{2} \times\right.\right. \\
&\left.\int_{0}^{\infty} \frac{d \epsilon_{d} \epsilon_{d}^{\frac{3}{2}} e^{\beta\left(\epsilon_{d}-\mu_{d}\right)}}{\left(e^{\beta\left(\epsilon_{d}-\mu_{d}\right)}+1\right)^{2}}-\frac{4}{3} \beta^{2} \int_{0}^{\infty} \frac{d \epsilon \epsilon^{\frac{3}{2}} e^{2 \beta\left(\epsilon_{d}-\mu_{d}\right)}}{\left(e^{\beta\left(\epsilon_{d}-\mu_{d}\right)}+1\right)^{3}}\right)+ \\
&\left.\frac{q_{D}^{4}}{(2 \pi)^{2}} \frac{(2 m)^{\frac{3}{2}}}{2 \hbar^{3}} \int_{0}^{\infty} \frac{d \epsilon_{d} \sqrt{\epsilon_{d}} e^{\beta\left(\epsilon_{d}-\mu_{d}\right)}}{\left(e^{\beta\left(\epsilon_{d}-\mu_{d}\right)}+1\right)^{2}}\right\}
\end{aligned}
$$

transforms the variables in all the integrands to $x=\beta\left(\epsilon_{d}-\mu_{d}\right)$ :

$$
\begin{aligned}
M^{\prime \prime}(T)= & \frac{1}{12 \pi^{3}} \frac{J^{2} V^{2} m^{2}}{N^{2} \hbar^{5} n q_{s}^{2}} \mu_{s}\left\{\frac { q _ { D } ^ { 6 } } { 8 \pi ^ { 2 } } \frac { \sqrt { 2 m \mu _ { s } } } { \hbar } \left(\frac{1}{\sqrt{\beta \mu_{s}}} \int_{-\beta \mu_{d}}^{\infty} d x \sqrt{x+\beta \mu_{d}} \frac{e^{x}}{\left(e^{x}+1\right)^{2}}+\right.\right. \\
& \left.\frac{2}{3} \frac{1}{\sqrt{\beta \mu_{s}}} \int_{-\beta \mu_{d}}^{\infty} d x\left(x+\beta \mu_{d}\right)^{\frac{3}{2}} \frac{e^{x}}{\left(e^{x}+1\right)^{2}}-\frac{4}{3} \frac{1}{\sqrt{\beta \mu_{s}}} \int_{-\beta \mu_{d}}^{\infty} d x\left(x+\beta \mu_{d}\right)^{\frac{3}{2}} \frac{e^{2 x}}{\left(e^{x}+1\right)^{3}}\right) \\
& \left.+\frac{q_{D}^{4}}{8 \pi^{2}} \frac{\left(2 m \mu_{s}\right)^{\frac{3}{2}}}{\hbar^{3}} \frac{1}{\left(\beta \mu_{s}\right)^{\frac{3}{2}}} \int_{-\beta \mu_{d}}^{\infty} d x \sqrt{x+\beta \mu_{d}} \frac{e^{x}}{\left(e^{x}+1\right)^{2}}\right\} .
\end{aligned}
$$

We write $\sqrt{2 m \mu_{s}}=\hbar q_{s}$ and $\left(2 m \mu_{s}\right)^{\frac{3}{2}}=\hbar^{3} q_{s}^{3}$. The above expression attains the form

$$
\begin{aligned}
M^{\prime \prime}(T)= & \frac{1}{12 \pi^{3}} \frac{J^{2} V^{2} m^{2}}{N^{2} \hbar^{5} n} \mu_{s}\left\{\frac { 1 } { 8 \pi ^ { 2 } } ( \frac { q _ { D } } { q _ { s } } ) ^ { 6 } q _ { s } ^ { 5 } \left(\frac{1}{\sqrt{\beta \mu_{s}}} \int_{-\beta \mu_{d}}^{\infty} d x \sqrt{x+\beta \mu_{d}} \frac{e^{x}}{\left(e^{x}+1\right)^{2}}+\right.\right. \\
& \left.\frac{2}{3} \frac{1}{\sqrt{\beta \mu_{s}}} \int_{-\beta \mu_{d}}^{\infty} d x\left(x+\beta \mu_{d}\right)^{\frac{3}{2}} \frac{e^{x}}{\left(e^{x}+1\right)^{2}}-\frac{4}{3} \frac{1}{\sqrt{\beta \mu_{s}}} \int_{-\beta \mu_{d}}^{\infty} d x\left(x+\beta \mu_{d}\right)^{\frac{3}{2}} \frac{e^{2 x}}{\left(e^{x}+1\right)^{3}}\right) \\
& \left.+\frac{1}{8 \pi^{2}}\left(\frac{q_{D}}{q_{s}}\right)^{4} \frac{q_{s}^{5}}{\left(\beta \mu_{s}\right)^{\frac{3}{2}}} \int_{-\beta \mu_{d}}^{\infty} d x \sqrt{x+\beta \mu_{d}} \frac{e^{x}}{\left(e^{x}+1\right)^{2}}\right\} .
\end{aligned}
$$

This is our final simplified expression(after implementing the above mentioned assumptions 1 and 2). Temperature dependence of the imaginary part of memory function gives the temperature dependence of resistivity $\rho(T)=\frac{m}{n e^{2}} \frac{1}{\tau(T)}=\frac{m}{n e^{2}} M^{\prime \prime}(T)$ [19, 20]. The expression (44) is plotted for various values of $\mu_{d}$ in figure (1a) and for various values of $q_{D}$ in figure (1b). We notice low temperature upturn (in (a) and (b)) and high temperature $T^{\frac{3}{2}}$ behaviour in figure (1c)(refer section 4.2 for details). 

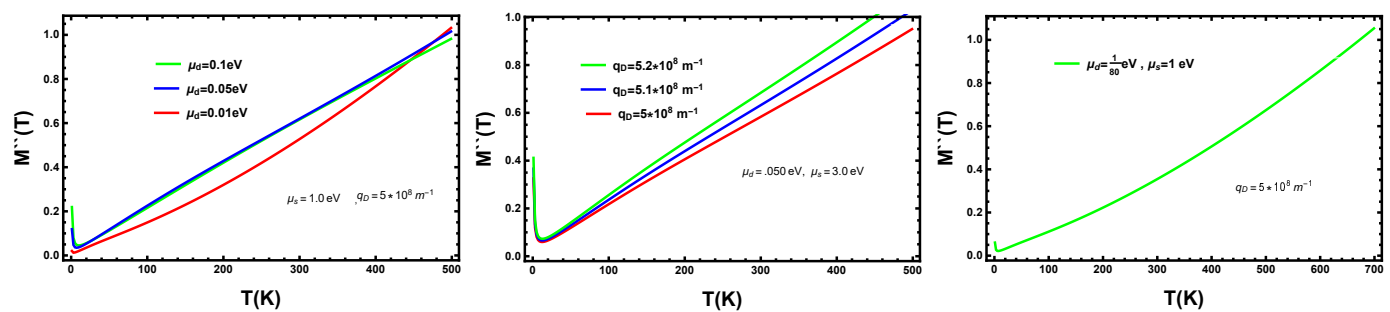

Figure 1: (a) $M^{\prime \prime}(T)$ for various values of $\mu_{d}$. (b) $M^{\prime \prime}(T)$ for various values of $q_{D}$. (c) High temp. behaviour of $M^{\prime \prime}(T) . M^{\prime \prime}(T) \propto T^{\frac{3}{2}}$ in high temperature limit $k_{B} T>>\mu_{d}$.

\section{Analysis of the general expression in special cases:}

\subsection{Low temperature limit $\left(k_{B} T<<\mu_{d}\right)$}

In this temperature limit we have $\beta \mu_{d}>>1$ thus the general expression (44) transforms to

$$
\begin{aligned}
M^{\prime \prime}(T) \simeq \frac{1}{12 \pi^{3}} & \frac{J^{2} V^{2} m^{2}}{N^{2} \hbar^{5} n} \mu_{s}\left\{\frac { 1 } { 8 \pi ^ { 2 } } ( \frac { q _ { D } } { q _ { s } } ) ^ { 6 } q _ { s } ^ { 5 } \left(\frac{\sqrt{\beta \mu_{d}}}{\sqrt{\beta \mu_{s}}} \int_{-\beta \mu_{d}}^{\infty} d x \frac{e^{x}}{\left(e^{x}+1\right)^{2}}+\frac{2}{3} \frac{\left(\beta \mu_{s}\right)^{\frac{2}{3}}}{\sqrt{\beta \mu_{s}}} \int_{-\beta \mu_{d}}^{\infty} d x \frac{e^{x}}{\left(e^{x}+1\right)^{2}}\right.\right. \\
& \left.-\frac{4}{3} \frac{\left(\beta \mu_{s}\right)^{\frac{2}{3}}}{\sqrt{\beta \mu_{s}}} \int_{-\beta \mu_{d}}^{\infty} d x \frac{e^{2 x}}{\left(e^{x}+1\right)^{3}}\right)+\frac{1}{8 \pi^{2}}\left(\frac{q_{D}}{q_{s}}\right)^{4} \frac{q_{s}^{5}}{\left(\beta \mu_{s}\right)^{\frac{3}{2}}} \sqrt{\beta \mu_{d}} \int_{-\beta \mu_{d}}^{\infty} d x \frac{e^{x}}{\left(e^{x}+1\right)^{2}},
\end{aligned}
$$

where we replaced $\sqrt{x+\beta \mu_{d}} \simeq \sqrt{\beta \mu_{d}}$ as $\beta \mu_{d}>>1$ and $x \sim 1$ due to exponentially damped function of the form $\frac{e^{x}}{\left(e^{x}+1\right)^{2}}$ in the integrands. With further rearrangements the above expression further simplifies to

$$
\begin{aligned}
& M^{\prime \prime}(T) \simeq \frac{1}{12 \pi^{3}} \frac{J^{2} V^{2} m^{2}}{N^{2} \hbar^{5} n} \mu_{s}\left\{\frac { 1 } { 8 \pi ^ { 2 } } ( \frac { q _ { D } } { q _ { s } } ) ^ { 6 } q _ { s } ^ { 5 } \left(\sqrt{\frac{\mu_{d}}{\mu_{s}}} \int_{-\beta \mu_{d}}^{\infty} d x \frac{e^{x}}{\left(e^{x}+1\right)^{2}}+\right.\right. \\
& \frac{2}{3} \frac{\left(\mu_{d}\right)^{\frac{3}{2}}}{\sqrt{\mu_{s}}} \frac{1}{k_{B} T} \int_{-\beta \mu_{d}}^{\infty} d x\left\{\frac{e^{x}}{\left(e^{x}+1\right)^{2}}-2 \frac{e^{2 x}}{\left(e^{x}+1\right)^{3}}\right\}+ \\
& \frac{1}{8 \pi^{2}}\left(\frac{q_{D}}{q_{s}}\right)^{4} \frac{q_{s}^{5} \sqrt{\mu_{d}}}{\left(\mu_{s}\right)^{\frac{3}{2}}} k_{B} T \int_{-\beta \mu_{d}}^{\infty} d x \frac{e^{x}}{\left(e^{x}+1\right)^{2}} .
\end{aligned}
$$

In the low temperature limit, the dominating term is the middle one with prefactor proportional to $\frac{1}{T}$. Neglecting the subdominating terms the memory function in low temperature limit reduces to

$$
M^{\prime \prime}(T \rightarrow 0) \sim \frac{1}{T} f_{s}(T), \quad f_{s}(T)=\int_{-\beta \mu_{d}}^{\infty} d x\left\{\frac{e^{x}}{\left(e^{x}+1\right)^{2}}-2 \frac{e^{2 x}}{\left(e^{x}+1\right)^{3}}\right\},
$$

where $f_{s}(T)$ is a slowly varying function iv of temperature. So, in the low temperature limit resistivity displays an upturn, as seen in figure (1a). An important point to be noted here is that the divergence in our case is of the form of power law instead of the logarithmic divergence in the original Kondo problem. The reason behind this difference is that we treated $\mathrm{d}$ or $\mathrm{f}$ electrons as quasi-localized (away from half-filling) instead of fully localized ones[1]. This is one of our important result.

\footnotetext{
${ }^{\text {iv }}$ We have checked the relative variation of $f_{s}(T)$ as compared to $\frac{1}{T}$ and found that relative variation of $f_{s}(T)$ is very small.
} 


\subsection{High temperature limit $\left(k_{B} T>>\mu_{d}\right)$}

In high temperature limit we have $\beta \mu_{d}<<1$. In this limit expression from (44) changes to

$$
\begin{gathered}
M^{\prime \prime}(T) \simeq \frac{1}{12 \pi^{3}} \frac{J^{2} V^{2} m^{2}}{N^{2} \hbar^{5} n} \mu_{s}\left\{\frac { 1 } { 8 \pi ^ { 2 } } ( \frac { q _ { D } } { q _ { s } } ) ^ { 6 } q _ { s } ^ { 5 } \left(\frac{1}{\sqrt{\beta \mu_{s}}} \int_{0}^{\infty} d x \sqrt{x} \frac{e^{x}}{\left(e^{x}+1\right)^{2}}+\right.\right. \\
\left.\frac{2}{3} \frac{1}{\sqrt{\beta \mu_{s}}} \int_{0}^{\infty} d x(x)^{\frac{2}{3}}\left[\frac{e^{x}}{\left(e^{x}+1\right)^{2}}-2 \frac{e^{2 x}}{\left(e^{x}+1\right)^{3}}\right]\right)+ \\
\left.\frac{1}{8 \pi^{2}}\left(\frac{q_{D}}{q_{s}}\right)^{4} \frac{q_{s}^{5}}{\left(\beta \mu_{s}\right)^{\frac{3}{2}}} \int_{0}^{\infty} d x \sqrt{x} \frac{e^{x}}{\left(e^{x}+1\right)^{2}}\right\} .
\end{gathered}
$$

By direct computation we notice that the last term in the above expression is many order of magnitude larger than the first two terms. Thus,

$$
\begin{aligned}
M^{\prime \prime}\left(k_{B} T>>\mu_{d}\right) \sim & C T^{\frac{3}{2}} \int_{0}^{\infty} d x \sqrt{x} \frac{e^{x}}{\left(e^{x}+1\right)^{2}} \sim 0.536 C T^{\frac{3}{2}} \\
& M^{\prime \prime}\left(k_{B} T>>\mu_{d}\right) \sim T^{\frac{3}{2}}
\end{aligned}
$$

where prefactor $C=\frac{1}{96 \pi^{5}} \frac{J^{2} V^{2} m^{2}}{N^{2} \hbar^{5} n}\left(\frac{q_{D}}{q_{s}}\right)^{4} \frac{q_{s}^{5}}{\sqrt{\mu_{s}}}$. Thus, in high temperature limit the memory function scales as $M^{\prime \prime}\left(k_{B} T>>\mu_{d}\right) \sim T^{\frac{3}{2}}$. This is also observed in figure (1c).

\section{Comparison with experimental data}

In this section we compare our theory with the experimental data. For comparison we consider Kondo-like behaviour observed in nano-scale granular aluminum samples [3]. Resistivity of

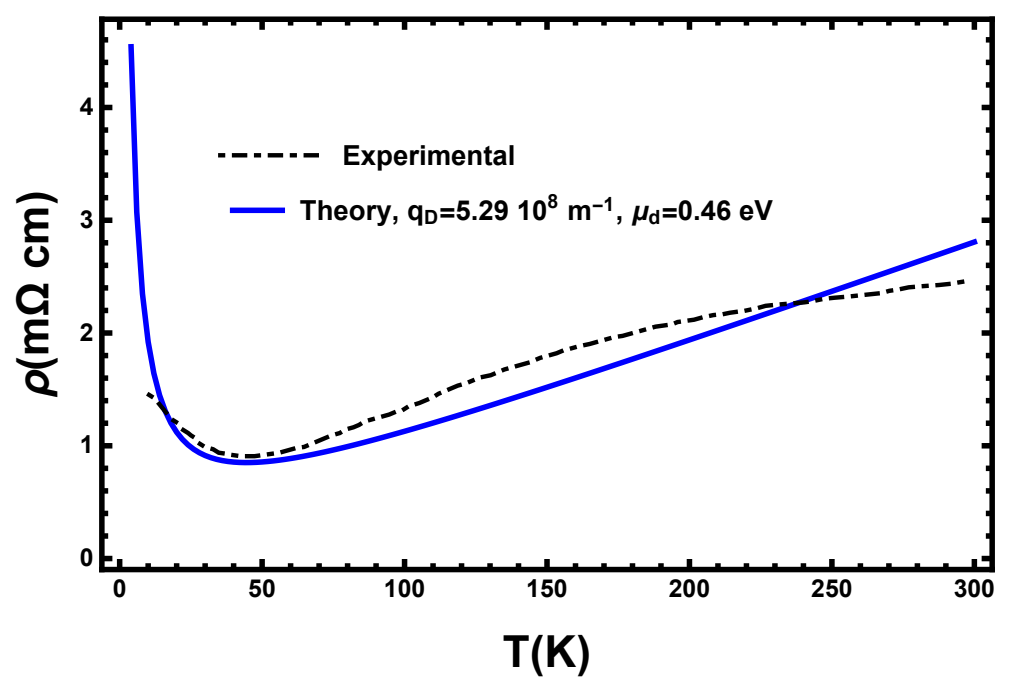

Figure 2: (a) Resistivity as a function of temperature compared with experimental data....

nano-scale granular aluminum samples was measured in reference[3]. Kondo-like behaviour was observed in the temperature dependence of resistivity. Resistivity shows low temperature upturn and a minimum around $T_{m} \simeq 40 K$, and then it shows negative curvature at higher temperature $\left(T>T_{m}\right)$. The experimental data in figure 2 of ref. [3] is reproduced here in figure 2 (dotted line). In the experimental paper it is argued that such a resistivity behaviour originates from spin-flip 
scattering of conduction electrons by local magnetic moments which are possibly located at the metal oxide interface. The physical explanation given in the experimental paper is reasonable as our theory is in good agreement with the data (figure 2). In the present theory we have spin-flip scattering of conduction electrons off the quasi-localized $d$ or $f$ electrons. The DC resistivity is computed using the present theory $\rho(T)=\frac{m}{n e^{2}} \frac{1}{\tau(T)}=\frac{m}{n e^{2}} M^{\prime \prime}(T)$ takes the form

$$
\begin{aligned}
\rho(T)= & \left(\frac{m}{n e^{2}}\right) \frac{1}{96 \pi^{5}} \frac{J^{2} V^{2} m^{2}}{N^{2} \hbar^{5} n} \mu_{s} q_{s}^{5}\left\{( \frac { q _ { D } } { q _ { s } } ) ^ { 6 } \left(\frac{1}{\sqrt{\beta \mu_{s}}} \int_{-\beta \mu_{d}}^{\infty} d x \sqrt{x+\beta \mu_{d}} \frac{e^{x}}{\left(e^{x}+1\right)^{2}}+\right.\right. \\
& \left.\frac{2}{3} \frac{1}{\sqrt{\beta \mu_{s}}} \int_{-\beta \mu_{d}}^{\infty} d x\left(x+\beta \mu_{d}\right)^{\frac{3}{2}} \frac{e^{x}}{\left(e^{x}+1\right)^{2}}-\frac{4}{3} \frac{1}{\sqrt{\beta \mu_{s}}} \int_{-\beta \mu_{d}}^{\infty} d x\left(x+\beta \mu_{d}\right)^{\frac{3}{2}} \frac{e^{2 x}}{\left(e^{x}+1\right)^{3}}\right) \\
& \left.+\frac{1}{8 \pi^{2}}\left(\frac{q_{D}}{q_{s}}\right)^{4} \frac{1}{\left(\beta \mu_{s}\right)^{\frac{3}{2}}} \int_{-\beta \mu_{d}}^{\infty} d x \sqrt{x+\beta \mu_{d}} \frac{e^{x}}{\left(e^{x}+1\right)^{2}}\right\}
\end{aligned}
$$

and it does show an up-turn at lower temperature, and as the temperature is raised it passes through a minima $\left(T_{m} \simeq 38 \mathrm{~K}\right)$ and then increase monotonically (figure 2 ). For the comparison of theory and experiment we take $\mu_{s}=11.5 \mathrm{eV}$, and lattice constant $a=4.05 \AA$ (both for metal Aluminum). We take $q_{D}$ and $\mu_{d}$ as our fitting parameters. The best fit value is obtained for $q_{D}=5.29 \times 10^{8} \mathrm{~m}^{-1}$ and $\mu_{d}=0.46 \mathrm{eV}$. From the figure 2, it is clear that the theory developed here is in reasonable agreement with the experimental data. By comparing the magnitudes of $\mu_{d} \simeq 0.46 \mathrm{eV}$ and $\mu_{s} \simeq 11.5 \mathrm{eV}$ we notice that the s-electrons form a bigger Fermi surface, and d-electrons form a smaller Fermi surface, as expected from our theoretical considerations.

\section{Conclusion}

The calculation of DC resistivity through the calculation of the memory function formalism $\left(\rho(T)=\frac{m}{n e^{2}} \frac{1}{\tau(T)}=\frac{m}{n e^{2}} M^{\prime \prime}(T)\right)$ for the Kondo lattice Hamiltonian (or $s$ - $d$ Hamiltonian) is presented. We used the Wölfle-Götze approximation to compute the memory function. The scattering of conduction electrons via the quasi-localized $f$ or $d$ electrons is taken into account by treating the $H_{s-d}$ part of Hamiltonian as a perturbation. Dispersion of spin excitations is taken to be of the form $\hbar \omega_{q}=c_{m} q^{2}$. We find that the D.C. resistivity shows low temperature $\left(k_{B} T<<\mu_{d}\right)$ power law up-turn and high temperature $\left(k_{B} T>>\mu_{d}\right) T^{\frac{3}{2}}$ scaling.

\section{Appendices:}

\section{A Average of spin density operators of localized elec- trons}

The commutator of spin density operators is written as:

$$
\begin{aligned}
\sum_{k^{\prime} k}\left\langle\left[S^{-}\left(k^{\prime}-k\right), S^{+}\left(k-k^{\prime}\right)\right]\right\rangle & =\left\langle\left[S^{-}(q), S^{+}(-q)\right]\right\rangle \\
& =\sum_{k}\left\langle\left[a_{k^{\prime} \downarrow}^{\dagger} a_{k \uparrow}, a_{k \uparrow}^{\dagger} a_{k^{\prime} \downarrow}\right]\right\rangle
\end{aligned}
$$

Here we set $k^{\prime}-k=q$, and to treat $d$ electrons as quasi-localized we write $S^{+}$and $S^{-}$in terms of Fermi functions $\left(S^{-}(q)=\sum_{k} a_{k+q \downarrow}^{*} a_{k \uparrow}\right)$. The anticommutation property simplifies the eqn 
(51) to

$$
\begin{aligned}
\sum_{k^{\prime} k}\left\langle\left[S^{-}\left(k^{\prime}-k\right), S^{+}\left(k-k^{\prime}\right)\right]\right\rangle & =\sum_{k, q}\left\langle a_{k^{\prime} \downarrow}^{\dagger}\left\{a_{k \uparrow}, a_{k \uparrow}^{\dagger}\right\} a_{k^{\prime} \downarrow}\right\rangle-\left\langle a_{k \uparrow}^{\dagger}\left\{a_{k^{\prime} \downarrow}^{\dagger}, a_{k^{\prime} \downarrow}\right\} a_{k \uparrow}\right\rangle \\
& =\sum_{k, q}\left(f_{k+q \downarrow}^{d}-f_{k \uparrow}^{d}\right)
\end{aligned}
$$

We use $f_{k^{\prime} \downarrow}^{d}=\left\langle a_{k^{\prime} \downarrow}^{\dagger} a_{k^{\prime} \downarrow}\right\rangle$ notation to differentiate Fermi function of $d$-band electrons from that of $s$-band electrons. The other factor in eqn (15) is:

$$
\begin{aligned}
\sum_{k^{\prime} k}\left\langle S^{+}\left(k-k^{\prime}\right) S^{-}\left(k^{\prime}-k\right)\right\rangle & =\left\langle S^{+}(-q) S^{-}(q)\right\rangle=\sum_{k+q}\left\langle a_{k \uparrow}^{\dagger} a_{k+q \downarrow} a_{k+q \downarrow}^{\dagger} a_{k \uparrow}\right\rangle \\
& =\sum_{k, q} f_{k \uparrow}^{d}\left(1-f_{k+q \downarrow}^{d}\right) .
\end{aligned}
$$

\section{B $\theta$ integral solution}

In the presence of Fermi factors of the form $f_{k^{\prime}}^{s}\left(1-f_{k}^{s}\right)$ and at ordinary temperature $k_{B} T<<$ $\mu_{s}(\sim \mathrm{eV})$, one can replace $\epsilon$ and $\epsilon^{\prime}$ inside the square root by $\mu_{s}$ for $s$ electrons $\left(\mu_{s}=\frac{\hbar^{2} q_{s}^{2}}{2 m}\right)$ where $q_{s}$ is Fermi wavevector for $s$-electrons:

$$
\begin{aligned}
\int_{0}^{\pi} \sin \theta d \theta \delta\left(q-\sqrt{2 m} \sqrt{\left(\epsilon^{\prime}+\epsilon-2 \sqrt{\epsilon^{\prime}} \cos \theta\right)}\right) & \simeq \int_{0}^{\pi} \sin \theta d \theta \delta(q-2 \sqrt{m \epsilon(1-\cos \theta)}) \\
& \simeq \int_{0}^{\pi} \sin \theta d \theta \delta\left(q-\sqrt{2} q_{s} \sqrt{(1-\cos \theta)}\right)
\end{aligned}
$$

Put $x=1-\cos \theta$ and define $\xi=q_{s} \sqrt{2 x}$ and the limit of the integral changes to 0 and $2 q_{s}$ (note that $0<q<q_{s}$ ). The integral becomes

$$
\int_{0}^{\pi} \sin \theta d \theta \delta\left(q-\sqrt{2 m} \sqrt{\left(\epsilon^{\prime}+\epsilon-2 \sqrt{\epsilon^{\prime}} \epsilon \cos \theta\right)}\right) \simeq \int_{0}^{2 k_{s}} \frac{\xi d \xi}{q_{s}^{2}} \delta(q-\xi) \simeq \frac{q}{q_{s}^{2}} .
$$

\section{Expansion of $f_{d}^{1}(q)$}

$$
f_{d}^{1}(q)=\sum_{k_{d}}\left[f^{d}\left(\epsilon_{k_{d}}\right)-f^{d}\left(\epsilon_{k_{d}^{\prime}}\right)\right]
$$

The Taylor's expansion for small $(q \rightarrow 0)$ gives

$$
f_{d}^{1}(q)=\sum_{k_{d}}\left[f^{d}\left(\epsilon_{k_{d}}\right)-f^{d}\left(\epsilon_{k_{d}}\right)-\left.q \frac{\partial f^{d}\left(\epsilon_{k_{d}^{\prime}}\right)}{\partial q}\right|_{q=0}-\left.\frac{q^{2}}{2 !} \frac{\partial^{2} f^{d}\left(\epsilon_{k_{d}^{\prime}}\right)}{\partial q^{2}}\right|_{q=0}-\left.\frac{q^{3}}{3 !} \frac{\partial^{3} f^{d}\left(\epsilon_{k_{d}^{\prime}}\right)}{\partial q^{3}}\right|_{q=0} \ldots\right] .
$$


on converting summation into integrals, we get

$$
f_{d}^{1}(q)=-\frac{V}{(2 \pi)^{2}} \int_{0}^{\infty} k_{d}^{2} d k_{d} \int_{0}^{\pi} \sin \theta d \theta\left[\left.q \frac{\partial f^{d}\left(\epsilon_{k_{d}^{\prime}}\right)}{\partial q}\right|_{q=0}+\left.\frac{q^{2}}{2 !} \frac{\partial^{2} f^{d}\left(\epsilon_{k_{d}^{\prime}}\right)}{\partial q^{2}}\right|_{q=0}+\left.\frac{q^{3}}{3 !} \frac{\partial^{3} f^{d}\left(\epsilon_{k_{d}^{\prime}}\right)}{\partial q^{3}}\right|_{q=0} \ldots\right]
$$

We have Fermi function $f^{d}\left(\epsilon_{k_{d}^{\prime}}, \theta\right)=\frac{1}{e^{\left.\beta \frac{\hbar^{2} q^{2}}{2 m}+\frac{\hbar^{2} k_{d}^{2}}{2 m}+\frac{\hbar^{2} k_{d} q \cos \theta}{m}-\mu_{d}\right]}+1}$. For simplification, we put $\alpha=$ $\beta\left(\frac{\hbar^{2} k_{d}^{2}}{2 m}-\mu_{d}\right), \eta=\beta \frac{\hbar^{2}}{2 m}$ and $\gamma=\beta \frac{\hbar^{2} k_{d}}{m}$. The Fermi function set to

$$
f^{d}(q, \alpha, \eta, \gamma, \theta)=\frac{1}{e^{\left[\alpha+\eta q^{2}+\gamma q \cos \theta\right]}},\left.\quad \frac{\partial f^{d}(\alpha, \gamma, \theta)}{\partial q}\right|_{q=0}=-\frac{e^{\alpha} \gamma \cos \theta}{\left(e^{\alpha}+1\right)^{2}},
$$

similarly

$$
\left.\frac{\partial^{2} f^{d}(\alpha, \eta, \gamma, \theta)}{\partial q^{2}}\right|_{q=0}=-\frac{e^{\epsilon_{d}-\mu_{d}}}{\left(e^{\epsilon_{d}-\mu_{d}}+1\right)^{2}}\left[2 \eta+\gamma^{2} \cos ^{2} \theta\right]+\frac{2 \gamma^{2} e^{\beta\left(\epsilon_{d}-\mu_{d}\right)} \cos ^{2} \theta}{\left(e^{\epsilon_{d}-\mu_{d}}+1\right)^{3}}
$$

the third derivative becomes

$$
\left.\frac{\partial^{3} f^{d}(\alpha, \eta, \gamma, \theta)}{\partial q^{3}}\right|_{q=0}=\frac{12 \eta e^{2 \alpha} \gamma \cos \theta}{\left(e^{\alpha}+1\right)^{3}}-\frac{6 \eta e^{\alpha} \gamma \cos \theta}{\left(e^{\alpha}+1\right)^{2}}-\frac{6 e^{3 \alpha} \gamma^{3} \cos ^{3} \theta}{\left(e^{\alpha}+1\right)^{4}}+\frac{6 e^{2 \alpha} \gamma^{3} \cos ^{3} \theta}{\left(e^{\alpha}+1\right)^{3}}-\frac{e^{\alpha} \gamma^{3} \cos ^{3} \theta}{\left(e^{\alpha}+1\right)^{2}},
$$

We substitute derivative terms of $f^{d}\left(\epsilon_{k_{d}^{\prime}}\right)$ from eqn (59), (160) and (61) in the expression (58) and perform $\theta$ integration. Thus replacing $\alpha, \eta$ and $\gamma$ with their respective terms we obtain

$$
f_{d}^{1}\left(\epsilon_{d}\right)=V \frac{q^{2}}{4 \pi^{2}} \frac{\sqrt{2 m}}{\hbar}\left[\beta \int_{0}^{\infty} \frac{d \epsilon_{d} \sqrt{\epsilon_{d}} e^{\beta\left(\epsilon_{d}-\mu_{d}\right)}}{\left(e^{\beta\left(\epsilon_{d}-\mu_{d}\right)}+1\right)^{2}}+\frac{2}{3} \beta^{2} \int_{0}^{\infty} \frac{d \epsilon_{d} \epsilon_{d}^{\frac{3}{2}} e^{\beta\left(\epsilon_{d}-\mu_{d}\right)}}{\left(e^{\beta\left(\epsilon_{d}-\mu_{d}\right)}+1\right)^{2}}-\frac{4}{3} \beta^{2} \int_{0}^{\infty} \frac{d \epsilon \epsilon^{\frac{3}{2}} e^{2 \beta\left(\epsilon_{d}-\mu_{d}\right)}}{\left(e^{\beta\left(\epsilon_{d}-\mu_{d}\right)}+1\right)^{3}}\right] .
$$

\section{Term $f_{d}^{2}(q)$ expansion}

The Fermi function of $d$-band electrons $f_{d}^{2}(q)$ is

$$
f_{d}^{2}(q)=\sum_{k_{d}} f^{d}\left(\epsilon_{k_{d}}\right)\left(1-f^{d}\left(\epsilon_{k_{d}^{\prime}}\right)\right.
$$

The Taylor's expansion for small $q$ expands the Fermi function in the form

$$
\begin{aligned}
f_{d}^{2}(q) & =\sum_{k_{d}} f^{d}\left(\epsilon_{k_{d}}\right)\left(1-f^{d}\left(\epsilon_{k_{d}}\right)-\left.q \frac{\partial f^{d}\left(\epsilon_{k_{d}^{\prime}}\right)}{\partial q}\right|_{q=0}-\left.\frac{q^{2}}{2 !} \frac{\partial^{2} f^{d}\left(\epsilon_{k_{d}^{\prime}}\right)}{\partial q^{2}}\right|_{q=0}-\ldots\right) \\
& =\sum_{k_{d}}\left[f^{d}\left(\epsilon_{k_{d}}\right)\left(1-f^{d}\left(\epsilon_{k_{d}}\right)\right)-\left.q f^{d}\left(\epsilon_{k_{d}}\right) \frac{\partial f^{d}\left(\epsilon_{k_{d}^{\prime}}\right)}{\partial q}\right|_{q=0}-\left.\frac{q^{2}}{2 !} f^{d}\left(\epsilon_{k_{d}}\right) \frac{\partial^{2} f^{d}\left(\epsilon_{k_{d}^{\prime}}\right)}{\partial q^{2}}\right|_{q=0}-\ldots\right] .
\end{aligned}
$$

On converting sum into integration

$$
\begin{aligned}
f_{d}^{2}\left(\epsilon_{k_{d}}\right)=V\left[\frac{1}{(2 \pi)^{2}} \int_{0}^{\infty} k_{d}^{2} d k_{d} f^{d}\left(\epsilon_{k_{d}}\right)\left(1-f^{d}\left(\epsilon_{k_{d}}\right)\right) \int_{0}^{\pi} \sin \theta d \theta-\frac{q^{2}}{2 !(2 \pi)^{2}} \int_{0}^{\infty} k_{d}^{2} d k_{d} f^{d}\left(\epsilon_{k_{d}}\right) \times\right. \\
\\
\left.\left.\int_{0}^{\pi} \sin \theta d \theta \frac{\partial^{2} f^{d}\left(\epsilon_{k_{d}^{\prime}}\right)}{\partial q^{2}}\right|_{q=0}-\ldots . .\right],
\end{aligned}
$$


which can further be written in terms of energy

$$
\begin{gathered}
f_{d}^{2}\left(\epsilon_{k_{d}}\right)=\frac{V}{(2 \pi)^{2}} \frac{(2 m)^{\frac{3}{2}}}{\hbar^{3}} \int_{0}^{\infty} \frac{d \epsilon_{d} \sqrt{\epsilon_{d}} e^{\beta\left(\epsilon_{d}-\mu_{d}\right)}}{\left(e^{\beta\left(\epsilon_{d}-\mu_{d}\right)}+1\right)^{2}}+\frac{V q^{2}}{4 \pi^{2}} \frac{\sqrt{2 m}}{\hbar}\left[\beta \int_{0}^{\infty} \frac{d \epsilon_{d} \sqrt{\epsilon_{d}} e^{\beta\left(\epsilon_{d}-\mu_{d}\right)}}{\left(e^{\beta\left(\epsilon_{d}-\mu_{d}\right)}+1\right)^{2}}+\right. \\
\left.\frac{2}{3} \beta^{2} \int_{0}^{\infty} \frac{d \epsilon_{d} \epsilon_{d}^{\frac{3}{2}} e^{\beta\left(\epsilon_{d}-\mu_{d}\right)}}{\left(e^{\beta\left(\epsilon_{d}-\mu_{d}\right)}+1\right)^{2}}-\frac{4}{3} \beta^{2} \int_{0}^{\infty} \frac{d \epsilon \epsilon^{\frac{3}{2}} e^{2 \beta\left(\epsilon_{d}-\mu_{d}\right)}}{\left(e^{\beta\left(\epsilon_{d}-\mu_{d}\right)}+1\right)^{3}}\right] .
\end{gathered}
$$

\section{References}

[1] Patrik Fazekas, "Lecture Notes On Electron Correlation and Magnetism", World Scientific (1999).

[2] Yi-Feng Yang, Zachary, Han-Oh Lee, J.D. Thompson and David Pines, Nature Vol. 454, 611-613 (2008).

[3] N. Bachar, S. Lerer, S. Hacohen-Gourgy, B. Almog, and G. Deutscher, Physical Review B 87, 214512 (2013).

[4] C. Pfleiderer, Rev. Mod. Phys. 81, 1551 (2009).

[5] Alex C Hewson and Jun Kondo, Scholarpedia, 4(3):7529 (2009).

[6] Jun Kondo, Progress of Theoretical Physics 32: 37 (1964).

[7] A.C. Hewson, "The Kondo Problem to Heavy Fermions", Cambridge, (1997).

[8] Vijay B. Shenoy "SERC School on Magnetism and Superconductivity"(2006) (http://www.physics.iisc.ernet.in/ shenoy/LectureNotes/kondo.pdf).

[9] Tadao Kasuya, Progress of Theoretical Physics, Vol. 16, No 1, July (1956).

[10] Isao Mannari, Progress of Theoretical Physics, Vol. 22, No 3, September (1959).

[11] R.J. Weiss and A.S. Marotta, J. Phys. Chem. Solids Pergamon Press Vol. 9 pp. 302-308 (1959).

[12] J. Mathon, Proc. Roy. Soc. A. 306, 355-368 (1968).

[13] D.L.Mills, J. Phys. Chem. Solids, Vol. 34, pp. 679-686 (1973).

[14] Kazuo Ueda and Toru Moriya, Journal Of The Physical Society Of Japan, Vol. 39, No. 3, November (1975).

[15] Kazuo Ueda, Journal Of The Physical Society Of Japan, Vol. 43, No. 5, November (1977).

[16] Komal Kumari, Raman Sharma and Navinder Singh, Physics Letter A 2645-2651 (2019).

[17] J. M. Ziman, Electrons and phonons, OUP, Oxford (2001).

[18] R. Kubo, J. Phys. Soc. Jpn. 12, 570 (1957); Rep. Prog. Phys. 29, 255 (1966).

[19] Navinder Singh, "Electronic Transport Theories: from Weakly to Strongly Correlated Materials", CRC Press (2016).

[20] W. Götze and P. Wölfe, Phys. Rev. B. 6, 1226 (1972). 